Proceedings of the 1992 IREE

Intemational Conference on Robotics and Automation Nice, France - May 1992

\title{
A Recursive Method for Finding Revolute-Jointed Manipulator Singularities
}

\author{
Joel W. Burdick \\ Dept. of Mechanical Engineering \\ California Institute of Technology
}

\begin{abstract}
A geometric application of screw theory is used to develop a recursive algorithm for computing all singular configurations of revolute-jointed manipulators with arbitrary geometry and an arbitrary number of joints. The depth of the recursion is linear in the number of joints, $n$, while the computational burden is proportional to $2^{n-2}$. This method does not require explicit construction of the Jacobian matrix elements or a determinant operation. Further, the screw axis of the singular motion is determined at no additional cost. The bifurcations of this algorithm are also explored.
\end{abstract}

\section{Introduction}

Revolute-jointed manipulator singularities can be characterized by the zero set of a transcendental function, $\Phi(\bar{\theta})=0$, where $\bar{\theta}$ is the vector of manipulator joint angles. The primary contribution of this paper is a recursive algorithm which can symbolically determine the form of $\Phi$ or numerically generate the zero set, or critical point set ${ }^{\ddagger}$, of $\Phi=0$ for manipulators with an arbitrary number of joints and an arbitrary geometry. The method has the following advantages:

- Construction of the Jacobian matrix is unnecessary, nor is a determinant operation required.

- The method is recursive, leading to a simple computer implementation, especially for a large $n$.

- The direction of the screw axis of the singular configuration is determined with no additional cost. Conversely, all configurations which have a given singular screw axis can also be easily determined.

Many previous investigators have used screw theory to analyze manipulator singularities $[1,2,3,4,5]$. Singularities have also been considered as exterior and interior workspace boundaries $[6,7]$. Kohli and Hsu $[6,7]$ develop a recursive algorithm for determining the singularities of manipulators with wrists. Their algorithm provides some of the same results as Section 2. This paper physically motivates the development of a different recursion, does not require the construction of the Jacobian matrix, considers a more general class of manipulators and singularities (i.e. general screw singularities of spatial manipulators), and con-

The term "critical point eurface" and "critical value surface" will respectively denote the loci of aingular configurations in the joint apace and workspace. siders the bifurcations of the algorithm, which were not considered in $[6,7]$. Pai [8] has introduced the notion of generic singularities, which are related to the bifurcations discussed in this paper. The work in this paper is an extension of the author's work in [9].

Let denote a screw, which can be represented by six screw coordinates: $=[\vec{\omega}, \vec{V}]^{T} . \omega \in \mathbb{R}^{3}$ is a unit vector coincident with the screw axis. $\vec{V}=h \vec{\omega}+\vec{\rho} \times \vec{\omega}$ where $\vec{\rho}$ is a vector from the reference frame origin to any point on the screw axis and $h$ is the screw pitch. Screws $\$_{1}=\left[\vec{\omega}_{1}, \vec{V}_{1}\right]^{T}$ and $\$_{2}=\left[\vec{\omega}_{2}, \vec{V}_{2}\right]^{T}$ are said to be reciprocal if:

$$
\vec{\omega}_{1} \cdot \vec{V}_{2}+\vec{\omega}_{2} \cdot \vec{V}_{1}=0 \text {. }
$$

In a singular configuration, a manipulator end-effector can not twist along one or more screw axes. Thus:

Theorem: [1] In a singular configuration, all joint axis screws are reciprocal to the screw which represents the singular twist motion. We call this screw the singular screw.

This basic idea will be applied in the following sections. Because of length restrictions, this paper focuses on the algorithm for regional manipulators, and summarizes the highly analogous methodology for spatial manipulators. A more complete exposition can be found in [10].

\subsection{Singularities of Regional Manipulators}

Revolute-jointed regional manipulator singular configurations have a simple geometry $[2,8,9]$ :

Theorem: The (zero pitch) singular screw axis intersects each joint axis, possibly at infinity, and the end-effector.

Using this theorem, all possible singular screws (which characterize all singular configurations) can be generated by the following recursive procedure.

1. Start with all lines (potential singular screws) passing through the end-effector. There are $\infty^{2}$ such lines.

2. Constrain these lines to intersect joint axis $n$.

3. Constrain the resulting set of lines from step 2 to intersect joint axis $n-1$. 
4. Constrain the resulting set of lines from step 3 to intersect joint axis $n-2$.

$$
\vdots
$$

$n+1$. Constrain the set of lines from step $n$ to intersect joint axis 1.

This procedure can be implemented as follows. Let the Plücker coordinates $t$ of a line, which by the above procedure will become a singular screw axis, be denoted by $\left({ }^{k} \mathbf{P},{ }^{k} \mathbf{P}\right)$, where $k$ indicates that quantities are expressed in the $k^{\text {th }}$ link frame, $\mathcal{F}_{k}$. The singular screw will be reciprocal to the $j^{\text {th }}$ joint axis screw if:

$$
\mathbf{Q}_{j} \cdot \overline{\mathbf{P}}+\overline{\mathbf{Q}}_{j} \cdot \mathbf{P}=0 \quad j=1, \cdots, n
$$

where $Q_{j}$ and $\bar{Q}_{j}$ are the Plücker coordinates of the $j^{\text {th }}$ joint axis.

Step 1: Let $\mathcal{F}_{\text {ee }}$ be a frame with basis vectors parallel to those of $\mathcal{F}_{n}$, but with origin at the end-effector. In

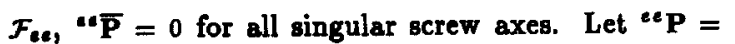
["e $P_{1}$, ${ }^{\text {ec }} P_{2}$,e $\left.P_{3}\right]^{T}$, where "e $P_{1}$, "e $P_{2}$, and ${ }^{e e} P_{3}$ satisfy the constraint: ${ }^{e e} P_{1}^{2}+{ }^{e e} P_{2}^{2}+{ }^{e e} P_{3}^{2}=1$.

Step 2: ${ }^{e e} Q_{n}=[0,0,1]^{T}$ and ${ }^{e} \bar{Q}_{n}=\left[0, a_{n}, 0\right]^{T}$. Condition (2.1), for $j=n$, is satisfied if ${ }^{\text {es }} P_{2}=0$.

Step 3: The Plücker coordinates of joint axis $n-1$ are:

$$
\begin{aligned}
& { }^{e} Q_{n-1}=\left[\begin{array}{lll}
s \theta_{n} s \alpha_{n-1}, & c \theta_{n} s \alpha_{n-1}, & c \alpha_{n-1}
\end{array}\right]^{T} \\
& * \bar{Q}_{n-1}=\left[\begin{array}{c}
d_{n} s \alpha_{n-1} c \theta_{n}+a_{n-1} c \alpha_{n-1} s \theta_{n} \\
-d_{n} s \alpha_{n-1} s \theta_{n}+c \alpha_{n-1}\left(a_{n-1} c \theta_{n}+a_{n}\right) \\
-\left(a_{n-1}+a_{n} c \theta_{n}\right) s \alpha_{n-1}
\end{array}\right]
\end{aligned}
$$

where $, \theta_{n}=\sin \theta_{n}, c \alpha_{n-1}=\cos \alpha_{n-1}$, etc. $a_{1} d$, and $\alpha$ are the standard Denavit-Hartenberg kinematic link parameters. Applying (2.1) and the constraint $\left.\right|^{e e} \mathbf{P} \mid=$ 1 results in:

$$
\text { "e } P_{1}=b_{1} / \sqrt{b_{1}^{2}+b_{2}^{2}} \quad \therefore P_{3}=b_{2} / \sqrt{b_{1}^{2}+b_{2}^{2}}
$$

where $b_{1}=\left(a_{n-1}+a_{n} c \theta_{n}\right) s \alpha_{n-1}$ and $b_{2}=d_{n} s \alpha_{n-1} c \theta_{n}+$ $a_{n-1} c \alpha_{n-1} s a_{n}$

Definition: Let the line which intersects axes $n-1, n$, and the end-effector be termed the $P_{0}$ tential Singular Screw(PSS) axis. For given $\theta_{n}$, the PSS axis is unique, except for degenerate cases discussed below.

Step 4: This is the first step in the recursion. Transform the Plücker coordinates of the PSS to $\mathcal{F}_{n-1}$ :

† 6 Plücker coordinater, ( $\left.p_{01}, p_{02}, p_{03}, p_{23}, p_{31}, p_{12}\right)$ parametrise a line, $\mathcal{L}$. $\left(p_{01}, p_{02}, p_{03}\right)=\mathbf{P}$ is a vector collinear to C. $\left(p_{23}, p_{31}, p_{12}\right)=\overline{\mathbf{P}}=\bar{\xi} \times \mathbf{P}$, where $\bar{\xi}$ is a vector from the origin of the reference frame Let to any point on $\mathcal{L}$. Lines with Plücker coordinates $\left(\mathbf{P}_{1}, \overline{\mathbf{P}}_{1}\right)$ and $\left(\mathbf{P}_{2}, \overline{\mathbf{P}}_{2}\right)$ intenect if $\mathbf{P}_{1} \cdot \overline{\mathbf{P}}_{2}+\overline{\mathbf{P}}_{1} \cdot \mathbf{P}_{2}=0$.

$$
\begin{gathered}
{ }^{n-1} P=\left[\begin{array}{c}
e e P_{1} c \theta_{n} \\
e e P_{1} c \alpha_{n-1} s \theta_{n}-e e P_{3} s \alpha_{n-1} \\
e e P_{1} s \alpha_{n-1} s \theta_{n}+{ }_{3} c \alpha_{n-1}
\end{array}\right] \\
{ }^{n-1} \bar{P}=\left[\begin{array}{c}
-e e P_{1} d_{n} s \theta_{n}+e e P_{3} a_{n} \Delta \theta_{n} \\
\left({ }^{e e} P_{1} d_{n}-a_{n} e e P_{3}\right) c \alpha_{n-1} c \theta_{n}-a_{n-1}{ }^{n-1} P_{3} \\
0
\end{array}\right]
\end{gathered}
$$

In a singular configuration, the singular screw axis must intersect joint axis $(n-1)$ at some distance $l_{n-1}$ from the origin of $\mathcal{F}_{n-1}$. Consequently ${ }^{n-1} \overline{\mathbf{P}}$ must have the form: ${ }^{n-1} \overline{\mathbf{P}}=\left[-\left(l_{n-1}{ }^{n-1} P_{2}\right),\left(l_{n-1}{ }^{n-1} P_{1}\right), 0\right]^{T}$. Thus, $l_{n-1}$ can be computed as:

$$
l_{n-1}=\frac{n-1 P_{2}}{n-1 P_{1}} ; \quad \text { or } \quad l_{n-1}=-\frac{n-1 P_{1}}{n-1 P_{2}}
$$

Algebraicly, $l_{n-1}=-a_{n-1} \tan \theta_{n} / s \alpha_{n-1}$.

The Plücker coordinates of joint axis $n-2$ are:

$$
\begin{aligned}
{ }^{n-1} Q_{n-2}= & {\left[s \theta_{n-1} s \alpha_{n-2}, c \theta_{n-1} s \alpha_{n-2}, c \alpha_{n-2}\right]^{T} } \\
{ }^{n-1} Q_{n-2}= & {\left[\begin{array}{c}
d_{n-1} \otimes \alpha_{n-2} c \theta_{n-1}+a_{n-2} c \alpha_{n-2} s \theta_{n-1} \\
-d_{n-1} \otimes \alpha_{n-2} s \theta_{n-1}+a_{n-2} c \alpha_{n-2} c \theta_{n-1} \\
-a_{n-2} s \alpha_{n-2}
\end{array}\right] }
\end{aligned}
$$

Substituting (2.4), (2.5), (2.7) into (2.1) results in:

$$
{ }^{n-1} K_{1} \cos \theta_{n-1}+{ }^{n-1} K_{2} \sin \theta_{n-1}+{ }^{n-1} K_{3}=0
$$

which can be expressed as a quadratic in $\tan \left(\frac{\theta_{n-1}}{2}\right)$ :

$$
\frac{{ }^{n-1} C_{1} \tan ^{2}\left(\frac{\theta_{n-1}}{2}\right)+2^{n-1} K_{2} \tan \left(\frac{\theta_{n-1}}{2}\right)+{ }^{n-1} C_{2}}{1+\tan ^{2}\left(\frac{\theta_{n-1}}{2}\right)}=0
$$

where ${ }^{n-1} C_{1}={ }^{n-1} K_{3}-^{n-1} K_{1},{ }^{n-1} C_{2}={ }^{n-1} K_{3}+{ }^{n-1} K_{1}$, and:

$$
\begin{aligned}
& { }^{n-1} K_{1}=\left(l_{n-1}+d_{n-1}\right) s \alpha_{n-2}{ }^{n-1} P_{1}+\left(a_{n-2} c \alpha_{n-2}\right)^{n-1} P_{2} \\
& { }^{=-1} K_{2}=\left(a_{n-2} c \alpha_{n-2}\right)^{n-1} P_{1}-\left(l_{n-1}+d_{n-1}\right) s \alpha_{n-2}{ }^{n-1} P_{2} \\
& { }^{n-1} K_{3}=-\left(a_{n-2} s \alpha_{n-2}\right)^{n-1} P_{3} .
\end{aligned}
$$

Assuming no roots at infinity or other nongeneric conditions, (2.8) has two roots:

$$
\tan \left(\frac{\theta_{n-1}}{2}\right)=\frac{-^{n-1} K_{2} \pm \sqrt{n-1} K_{1}^{2}+{ }^{n-1} K_{2}^{2}-{ }^{n-1} K_{3}^{2}}{n^{n-1} K_{3}-{ }^{n-1} K_{1}} .
$$

This step of the procedure has a simple geometric interpretation. Given $\theta_{n},(2.4)$ and (2.5) determine a unique (except in degenerate cases discussed below) line intersecting the end-effector and joint axes $n-1$, $n$. To create a singular configuration, the PSS is next rotated about joint axis $n-1$ until it intersects joint axis $n-2$. Geometrically, this rotation produces a double cone (with cone apex located at $l_{n-1}$ in $\mathcal{F}_{n-1}$ ) whose generator is the PSS axis. Generically, axis $n-2$ will have two intersections with the PSS cone. Infinite numbers of intersections can occur in nongeneric cases. 
In [9] it is shown that (2.8) is equivalent to $\operatorname{det}(\mathrm{J}(\bar{\theta}))=0$ for a 3R manipulator with arbitrary geometry.

Step j: The above algorithm can be recursively extended to 4 or more joints. If the intersection of the PSS axis with axis $j$ has been determined (to fix values of $\theta_{j+1}$ ), the coordinates of the PSS axis are transformed from $\mathcal{F}_{j+1}$ to $\mathcal{F}_{j}$ :

$$
\begin{aligned}
& { }^{j} \mathbf{P}={ }^{j} \mathbf{R}^{j+1}{ }^{j+1} \mathbf{P} \\
& { }^{j} \overline{\mathbf{P}}=\mathbf{L}_{j, j+1} \times{ }^{j} \mathbf{P}+{ }^{j} \mathbf{R}^{j+1}{ }^{j+1} \mathbf{P}
\end{aligned}
$$

where $L_{j, j+1}=\left[a_{j},-d_{j+1} \cos \alpha_{j-1}, d_{j+1} \sin \alpha_{j}\right]^{T}$ and ${ }^{j} \mathbf{R}^{j+1}$, which relates the orientation of $\mathcal{F}_{j+1}$ to $\mathcal{F}_{j}$, is evaluated with the value(s) of $\theta_{j+1}$ that ensure intersection of the PSS axis with axis $j$.

The PSS axis will intersect joint axis $j-1$ if (2.1) is satisfied, where ${ }^{j} \mathbf{Q}_{j-1}$ and ${ }^{j} \bar{Q}_{j-1}$ have the form of (2.7) with appropriate change of indices. This intersection condition has the form:

$$
{ }^{j} K_{1} \cos \theta_{j}+{ }^{j} K_{2} \sin \theta_{j}+K_{3}=0 .
$$

where: ${ }^{j} K_{1}$ has the same form as ${ }^{n-1} K_{1}$ in (2.9) with changes of indices $n-1 \rightarrow j$. Assuming non-generic conditions are not in effect, (2.12) also has a quadratic solution.

This recursion is continued until all joint angles $\left\{\theta_{2}, \cdots\right.$, $\left.\theta_{n-1}\right\}$ that lead to a singular configuration, for a given $\theta_{n}$, are found. Singular configurations are independent of $\theta_{1}$.

Remark: Genericly, for a given singular screw axis, there are up to $2^{n-2}$ related singular configurations in which all joint axis screws are reciprocal to the singular screw.

Examples of the critical point surfaces found using this algorithm are shown in Figure 1.

\section{Bifurcations of the Algorithm}

The critical point surfaces can be numerically traced by sweeping $\theta_{n}$ through the interval $[-\pi, \pi]$ and solving the above equations. Equivalently, this procedure traces the zero set of $n-2$ simultaneous equations (in this case quadratics of the form (2.12)):

$$
\begin{gathered}
G_{1}\left(\theta_{2}, \theta_{3}, \cdots, \theta_{n}\right)=0 \\
G_{2}\left(\theta_{3}, \cdots, \theta_{n}\right)=0 \\
\vdots \\
G_{n-2}\left(\theta_{n-1}, \theta_{n}\right)=0
\end{gathered} .
$$

where $G_{j}$ is equation (2.12) which is solved to find values of $\theta_{j+1}$ which insure intersection of the PSS axis with axis $j .(3.1)$ is of the form: $\mathbf{G}(\vec{x}, \lambda)=0$, where $\vec{x}=\left\{\theta_{2}, \cdots, \theta_{n-1}\right\}$ and $\lambda=\theta_{n}$. Finding the zero set of (3.1) is equivalent to studying the bifurcation of $G(\vec{x}, \lambda)=0$ with state vector $\vec{x}$ and bifurcation parameter $\lambda[11]$. The zero-sets of systems are subject to possible bifurcations, or self-intersections. These bifurcations must be considered for a robust implementation of this algorithm.

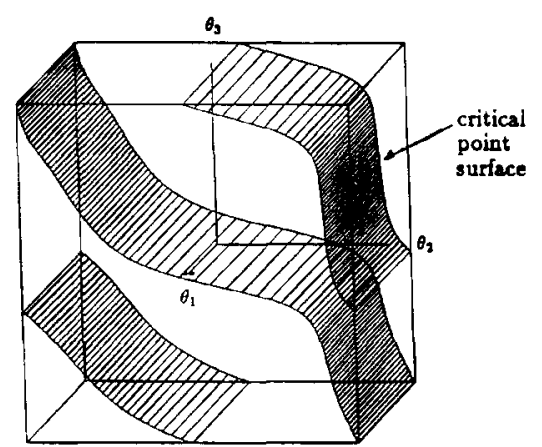

(a): Generic geometry: $\alpha_{1}=60^{\circ}, \alpha_{2}=-90^{\circ}$,

$a_{1}=1.3, \quad a_{2}=1.0, \quad a_{3}=1.6, d_{2}=0.4, d_{3}=-0.2$.

$\theta_{3}$

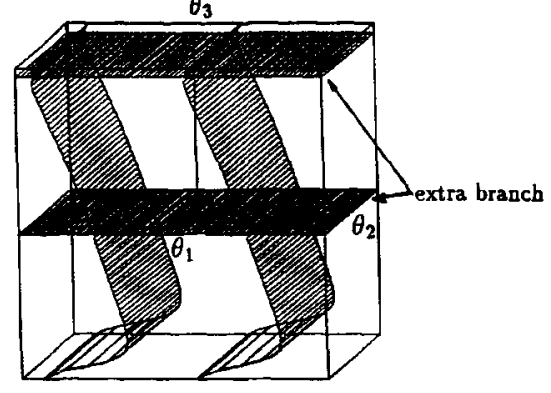

(b): Nongeneric geometry: $\alpha_{1}=60^{\circ}, \alpha_{2}=-45^{\circ}$, $a_{1}=0.0, \quad a_{2}=1.1, a_{3}=1.0, d_{2}=0.3, d_{3}=0.3$.

Figure 1: Generic and Non-Generic 3R Manipulator Critical Point Surfaces

Consider a $3 R$ manipulator. Note that if

$$
{ }^{2} K_{1}\left(\theta_{3}\right)={ }^{2} K_{2}\left(\theta_{3}\right)={ }^{2} K_{3}\left(\theta_{3}\right)=0
$$

any value of $\theta_{2}$ solves (2.10). Singularities arising from (3.2) are termed "extra branch" singularities [9]. The numerous geometric conditions which lead to (3.2) can be found in $[9,10]$. Figure 1 shows the critical point surfaces for a non-generic manipulator satisfying (3.2). When (3.2) is satisfied, a numerical algorithm based on a straightforward application of Section 2 would miss this extra branch of solutions.

(3.2) generalizes to $n R$ regional manipulators. Extra branches are present whenever ${ }^{j} K_{1}={ }^{j} K_{2}={ }^{j} K_{3}=0$. These bifurcations can be robustly detected as follows. In [11] it is proven that $G(\vec{x}, \lambda)=0$ bifurcates when $\operatorname{det}(\partial G(\vec{x}) / \theta \vec{x})$ changes sign. For $(3.1), \operatorname{det}(\theta G(\vec{x}) / \theta \vec{x})$ takes the form

$$
\operatorname{det}\left[\begin{array}{ccccc}
\frac{\partial G_{1}}{\partial \theta_{2}} & \frac{\partial G_{1}}{\partial \theta_{3}} & \cdots & \frac{\partial G_{3}}{\partial \theta_{n-2}} & \frac{\partial G_{1}}{\partial \theta_{n-1}} \\
0 & \frac{\partial G_{2}}{\partial \theta_{3}} & \cdots & \frac{\partial G_{2}}{\partial \theta_{n-2}} & \frac{\partial G_{2}}{\partial \theta_{n-1}} \\
0 & 0 & \cdots & \frac{\partial G_{3}}{\partial \theta_{n-2}} & \frac{\partial G_{3}}{\partial \theta_{n-1}} \\
\vdots & \vdots & \vdots & \vdots & \vdots \\
0 & 0 & \cdots & 0 & \frac{\partial G_{n-2}}{\partial \theta_{n-1}}
\end{array}\right]
$$


and thus bifurcation occurs when the product

$$
\frac{\partial G_{1}}{\partial \theta_{2}} \frac{\partial G_{2}}{\partial \theta_{3}} \cdots \frac{\partial G_{j}}{\partial \theta_{j+1}} \cdots \frac{\partial G_{n-2}}{\partial \theta_{n-1}}
$$

changes sign. (3.4) will change sign when any one of its components changes sign:

$$
\frac{\partial G_{j}}{\partial \theta_{j+1}}=-{ }^{j+1} K_{1} \sin \theta_{j+1}+{ }^{j+1} K_{2} \cos \theta_{j+1}
$$

While recursively solving (2.12), (3.4) and (3.5) can be easily computed. A change in sign from the previous increment in $\theta_{n}$ indicates that a bifurcation has occurred. Only one element in the product (3.4) typically changes sign. If $\delta G_{j} / \delta \theta_{j+1}$ changes sign, a bifurcation occurs in which all values of $\theta_{j+1}$ (for given $\left.\left\{\theta_{j}+2, \cdots, \theta_{n}\right\}\right)$ form an extra branch. Sweeping $\theta_{j+1}$ through $[-\pi, \pi]$ and recursively solving $(2.12)$ for $\left\{\theta_{2}\right.$, $\left.\cdots, \theta_{j}\right\}$ will trace out the extra branch(es).

The above algorithm assumes the PSS axis intersecting the end-effector and axes $n, n-1$ is unique for given $\theta_{n}$. There are three degenerate cases, termed "initial

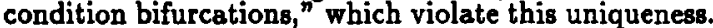
In each case there is a unique PSS axis for all $\theta_{n}$, except for one or two special values of $\theta_{n}$, where an infinite number of PSS axes arise. These extra branches of PSS axes can be parametrized by an angle $\phi \in[-\pi, \pi]$ as follows:

1. The last two axes intersect. When $\theta_{n}= \pm \pi / 2$, there are an infinity of PSS axes lying in the plane spanned by joint axes $n-1$ and $n$. The Plücker coordinates of the two extra branch PSS axes are: ${ }^{n-1} P=$ $[0, \cos \phi, \sin \phi]^{T} ;^{n-1} \overline{\mathbf{P}}=\left[ \pm a_{n} \sin \phi, 0,0\right]^{T}$.

2. The last two axes are parallel. The extra branches occur when $\theta_{n}=0, \pi$. In these case: ${ }^{n-1} \mathbf{P}=$ $[\cos \phi, 0, \sin \phi]^{T} ;^{n-1} \overline{\mathbf{P}}_{\theta_{x}=0}=\left[0, \cos \phi\left(d_{n}-\left(a_{n-1} \pm\right.\right.\right.$ $\left.\left.\left.a_{n}\right) \tan \phi\right), 0\right]^{T}$.

3. The end-effector intersects joint axis n-1 if $a_{n}^{2}=a_{n-1}^{2}+$ $d_{n} \tan ^{2} \alpha_{n-1}$ and $\theta_{n}=\operatorname{atan} 2\left(d_{n} \tan \alpha_{n-1} / a_{n}\right.$, $\left.-a_{n-1} / a_{n}\right)$. The Plücker coordinates of the extra branch PSS axes are ${ }^{n-1} P=\left(1 / a_{n}\right)\left[-a_{n-1} \cos \phi\right.$, $\sin \alpha_{n-1}\left(d_{n} \cos \phi-\sin \phi\right), d_{n} \tan \alpha_{n-1} \sin \alpha_{n-1} \cos \phi+$ $\left.\cos \alpha_{n-1} \sin \phi\right]$ and ${ }^{n-1} \overline{\mathbf{P}}=\left[0,-\left(d_{n} a_{n-1} \cos \phi\right) /\right.$ $\left.\left(a_{n} \cos \alpha_{n-1}\right), 0\right]^{T}$. There is one extra branch, except when cos $a_{n-1}=0$ and $a_{n} \geq a_{n-1}$, in which case there are two extra branches at $\theta_{n}= \pm(\pi / 2+$ $\left.\cos ^{-1}\left(a_{n-1} / a_{n}\right)\right)$. The Plücker coordinates of the PSS axes are: ${ }^{n-1} \mathbf{P}=\left[\begin{array}{lll}\cos \phi & \sin \phi\end{array}\right]^{T}$ and ${ }^{n-1} \overline{\mathbf{P}}=$ $\left[0 \pm \sqrt{a_{n}^{2}-a_{n-1}^{2}} 0\right]^{T}$

Thus, the complete critical point surfaces can be robustly traced for revolute-jointed regional manipulators with arbitrary joint numbers and arbitrary geometries by the following algorithm.

1. Set $\theta_{n}=-\pi ; j=n-1$

2. Compute: ${ }^{n-1} \mathbf{P}$ and ${ }^{n-1} \mathbf{P}$

3. Compute: ${ }^{j} K_{1},{ }^{j} K_{2},{ }^{j} K_{3}$.
4. Solve: ${ }^{j} K_{1} \cos \theta_{j}+{ }^{j} K_{2} \sin \theta_{j}+{ }^{j} K_{3}=0$ for $\left(\boldsymbol{\theta}_{j, 1}, \boldsymbol{\theta}_{j, 2}\right)$

5. Compute: $\operatorname{sgn}\left(\theta G_{j-1} / \partial \theta_{j}\right)$ for $\mathbf{k}=1,2$. If bifurcation, trace the extra branches.

6. If $j=1$, set $\theta_{n}=\theta_{n}+\Delta \theta_{n}$ and $j=n-1$; If $\theta_{n}<\pi$, go to step 2, else continue.

Else, transform the PSS axis from $\mathcal{F}_{j+1}$ to $\mathcal{F}_{j}$; set $j=j-1$; and go to step 3 .

7. If one of the degenerate PSS initial conditions occurs, trace the extra branches, as outlined above.

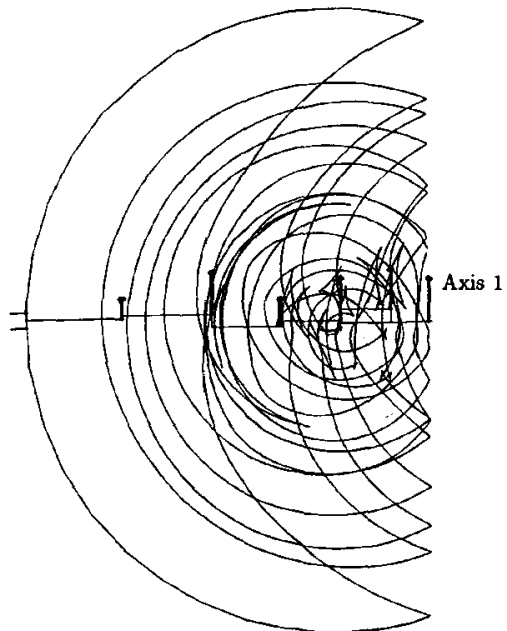

Figure 2: Cross Section of 6R Regional Singularities

$\Delta \theta_{n}$ can be chosen to be a small uniform constant. Alternatively, $\Delta \theta_{n}$ can be variable, with its magnitude chosen using step size bounding analyses [11]. Figure 2 shows a cross section, in the workspace, of the singular surfaces of a $6 \mathrm{R}$ manipulator (interpreted here as a redundant regional manipulator) with kinematic parameters $\alpha_{0}=0, \alpha_{1}=-70^{\circ}, \alpha_{2}=50^{\circ}, \alpha_{3}=60^{\circ}$ $\alpha_{4}=40^{\circ}, \alpha_{5}=-25^{\circ}, a_{0}=0, a_{1}=1.0, a_{2}=0.5$, $a_{3}=0.8, a_{4}=0.4, a_{5}=1.0, a_{6}=0.96, d_{1}=0, d_{2}=0.2$, $d_{3}=0.3, d_{4}=-0.1, d_{5}=0.4, d_{6}=0.15, d_{7}=0$

\section{Singularities of Spatial Manipulators}

The singular screw of a spatial manipulator singular configuration can have arbitrary pitch. The set of all possible singular screws is termed the singular serew field, $\mathcal{S}$. The corresponding set of singular configurations has been called the singularity field [5]. This section derives a recursive method, analogous to the method in Section 2, for determining $\mathcal{S}$, and thus all possible singular configurations. Spatial manipulator singular screws can be categorized by their pitch:

Rotational Singularities have singular screws with infinite pitch. There are $2^{n-2}$ such singularities which occur when when $\left\{\theta_{j}=0\right.$ or $\pi ; j=$ $2, \cdots, n-1\}$.

Regional Singularities have singular screws with zero pitch. 
General Screw Singularities have singular screws with a non-zero, but finite, pitch.

Rotational singular are trivial, and a more complete discussion can be found in [10], while the regional singularities can be determined using the method of Sections 2,3. Thus, we now focus a forward recursive algorithm for determining general screw singularities.

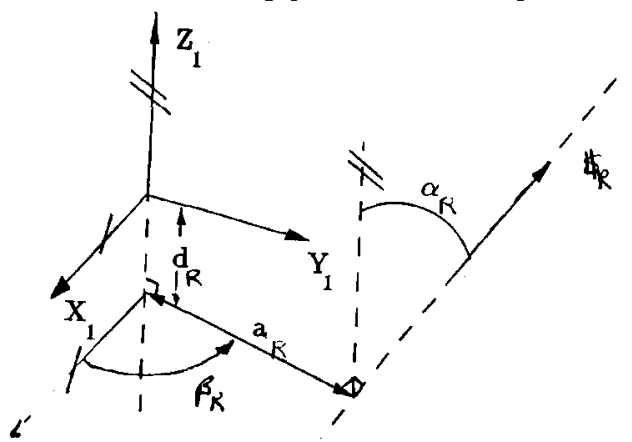

Figure 3: Parametrization of Singular Screw

Let a screw, $s_{R} \in S$ be parametrized by pitch h, and variables $\alpha_{R}, \beta_{R}, a_{R}$, and $\alpha_{R}$ (Fig. 3). The singularities of an $n R$ manipulator are independent of $\theta_{1}$, and $\$_{R}$ can be expressed in $\mathcal{F}_{1}$ coordinates. In $\mathcal{F}_{1}, \$_{R}$ will have the form $\$_{R}=\left[{ }^{1} V_{R}^{T},{ }^{1} \omega_{R}^{T}\right]^{T}$ :

$$
\begin{aligned}
{ }^{1} V_{R} & =\left[\begin{array}{c}
\left(h s \alpha_{R}+a_{R} c \alpha_{R}\right) s \beta_{R}+d_{R} s \alpha_{R} c \beta_{R} \\
-\left(h s \alpha_{R}+a_{R} c \alpha_{R}\right) c \beta_{R}+d_{R} s \alpha_{R} s \beta_{R} \\
h c \alpha_{R}-a_{R} s \alpha_{R}
\end{array}\right] \\
{ }^{1} \omega_{R} & =\left[\begin{array}{lll}
s \beta_{R} s \alpha_{R} & -c \beta_{R} s \alpha_{R} & c \alpha_{R}
\end{array}\right] .
\end{aligned}
$$

Note that in this parametrization, when $\alpha_{R}=0, d_{R}$ is not defined.

The singular screw parameters can be determined by consecutively constraining $\boldsymbol{s}_{R}$ to be reciprocal to the zero pitch joint axis screws: $\boldsymbol{s}_{j}=\left[\mathrm{V}_{j}^{T}, \bar{\omega}_{j}^{T}\right]^{T}$ :

$$
\mathbf{V}_{R} \cdot \bar{\omega}_{j}+\mathbf{V}_{j} \cdot \bar{\omega}_{R}=0 \quad(j=1, \cdots, n)
$$

where $\boldsymbol{s}_{j}$ denotes the screw coordinates of the $j^{\text {th }}$ joint axis. In $\mathcal{F}_{1}, \$_{1}$ and $\boldsymbol{s}_{2}$ have screw coordinates:

$$
\begin{aligned}
& s_{1}=\left[\begin{array}{llllll}
0 & 0 & 0 & 0 & 0 & 1
\end{array}\right] \\
& s_{2}=\left[\begin{array}{llllll}
0 & -a_{1} \cos \alpha_{1} & -a_{1} \sin \alpha_{1} & 0 & -\sin \alpha_{1} & \cos \alpha_{1}
\end{array}\right] .
\end{aligned}
$$

The application of (4.2) for $j=1,2$ yields:

$$
\begin{aligned}
& { }^{1} V_{R 3}=h \cos \alpha_{R}-a_{R} \sin \alpha_{R}=0 \\
& { }^{1} V_{R 2}=-a_{1}\left(\frac{{ }^{1} \omega_{R 2}}{\tan \alpha_{1}}+{ }^{1} \omega_{R 3}\right)
\end{aligned}
$$

where ${ }^{1} \omega_{R j}$ and ${ }^{1} V_{R j}(j=1,2,3)$ denote the $j^{\text {th }}$ elements of the vectors in (4.2). Of the $\infty^{5}$ possible screws in $\mathcal{F}_{1}$, only $\infty^{3}$ can be reciprocal to joint axes 1 and 2 . Hence, $S$ consists of not more than $\infty^{3}$ screws. This result has previously been established in [5].
Any three of the five singular screw parameters can be chosen to parametrize $\mathcal{S}$. Choose $h, \alpha_{R}$, and $\beta_{R}$. Using (4.3), the dependent parameters $a_{R}$ and $d_{R}$ are:

$$
\begin{aligned}
& a_{R}=h / \tan \alpha_{R} \\
& d_{R}=\frac{h}{\tan \beta_{R} \sin ^{2} \alpha_{R}}+\frac{a_{1}}{s \beta_{R}}\left(\frac{c \alpha_{R} c \beta_{R}}{s \alpha_{1}}-\frac{c \alpha_{R}}{s \beta_{R}}\right)
\end{aligned}
$$

By varying $\alpha_{R}, \beta_{R}$, and $h$ through their respective ranges, all possible $\infty^{3}$ elements of $S$ are generated. $\alpha_{R} \in(0, \pi / 2]$ for $h>0$ and $\alpha_{R} \in[-\pi / 2,0)$ if $h<0$. $\beta_{R} \in[0,2 \pi]$.

Consider one screw, $s_{R}^{*} \in S$ (e.g., fix $h, \alpha_{R}$, and $\beta_{R}$ ). The singular configurations in which the joint axis screws are reciprocal to $\boldsymbol{s}_{R}^{*}$ can be determined by recursively applying (4.2). First apply (4.2) to find the value(s) of $\theta_{2}$ which ensure reciprocity between joint axis 3 and $s_{R}^{*}$. In $\mathcal{F}_{1}, s_{3}=\left[{ }^{1} \mathbf{V}_{3}^{T},{ }^{1} \bar{\omega}_{3}^{T}\right]^{T}$, where:

$$
\begin{aligned}
{ }^{1} \mathrm{~V}_{3}= & {\left[\begin{array}{c}
d_{2} s \alpha_{1} c \theta_{2}+\alpha_{2} c \alpha_{2} s \theta_{2} \\
\eta c \alpha_{1}+\xi s \alpha_{1} \\
\eta s \alpha_{1}-\xi c \alpha_{1}
\end{array}\right] } \\
{ }^{1} \bar{\omega}_{3}= & {\left[\begin{array}{c}
s \alpha_{2} s \theta_{2} \\
-\left(c \theta_{2} c \alpha_{1} s \alpha_{2}+s \alpha_{1} c \alpha_{2}\right) \\
-c \theta_{2} s \alpha_{1} s \alpha_{2}+c \alpha_{1} c \alpha_{2}
\end{array}\right] . }
\end{aligned}
$$

and $\eta=d_{2} s \alpha_{2} s \theta_{2}-\left(a_{1}+a_{2} c \theta_{2}\right) c \alpha_{2} ; \xi=\left(a_{1} c \theta_{2}+a_{2}\right) s \alpha_{2}$. Substituting (4.1) and (4.5) into (4.2) yields the following condition for reciprocity: where:

$$
{ }^{1} D_{1} \cos \theta_{2}+{ }^{1} D_{2} \sin \theta_{2}+{ }^{1} D_{3}=0 ;
$$

$$
\begin{aligned}
{ }^{1} D_{1} & ={ }^{1} \omega_{R 1}\left(d_{2} s \alpha_{2}\right)+{ }^{1} \omega_{R 2}\left(a_{1} s \alpha_{1} s \alpha_{2}-a_{2} c \alpha_{1} c \alpha_{2}\right) \\
& -{ }^{1} \omega_{R 3}\left(a_{1} c \alpha_{1} s \alpha_{2}+a_{2} s \alpha_{1} c \alpha_{2}\right)-{ }^{1} V_{R 2}\left(c \alpha_{1} s \alpha_{2}\right) \\
{ }^{1} D_{2} & ={ }^{1} \omega_{R 1}\left(a_{2} c \alpha_{2}\right)+{ }^{1} \omega_{R 2}\left(d_{2} c \alpha_{1} s \alpha_{2}\right) \\
& +{ }^{1} \omega_{R 3}\left(d_{2} s \alpha_{1} s \alpha_{2}\right)+{ }^{1} V_{R 1} s \alpha_{2} \\
{ }^{1} D_{3} & ={ }^{1} \omega_{R 2}\left(-a_{1} c \alpha_{1} c \alpha_{2}+a_{2} s \alpha_{1} s \alpha_{2}\right)- \\
& { }^{1} \omega_{R 3}\left(a_{1} s \alpha_{1} c \alpha_{2}+a_{2} c \alpha_{1} s \alpha_{2}\right)-{ }^{1} V_{R 2} s \alpha_{1} c \alpha_{2}
\end{aligned}
$$

and the values of ${ }^{1} \omega_{R j}$ and ${ }^{1} V_{R j}$ assume that the constraints in (4.3) are active. (4.6) can be converted to a quadratic in $\tan \left(\theta_{2} / 2\right)$. Assuming non-generic bifurcations are not in effect, there are two values of $\theta_{2}$ which position axis 3 so that it is reciprocal to $s_{R}^{*}$. This is highly analogous to the algorithm derived in Sections 2,3 .

The values of $\theta_{3}$ which cause joint axis 4 to be reciprocal to $\boldsymbol{s}_{R}^{*}$ can be similarly determined. First, reexpress the potential singular screw axis in $\mathcal{F}_{2}$ :

$$
\begin{aligned}
{ }^{2} \bar{\omega}_{R} & =\left({ }^{1} R^{2}\right)^{T}{ }^{1} \bar{\omega}_{R} \\
{ }^{2} V_{R} & =\left({ }^{1} R^{2}\right)^{T}{ }^{1} V_{R}+\left({ }^{1} R^{2}{ }^{T} L_{1,2}\right) \times{ }^{2} \bar{\omega}_{R}
\end{aligned}
$$

where ${ }^{1} \mathbf{R}^{2}$ incorporates the values of $\theta_{2}$ which solve (4.6); and $L_{1,2}$ is the same as (2.11). Next, solve (4.6) 
(with appropriate change of index) for two values of $\theta_{3}$. Continuing, the values of $\theta_{4}, \cdots, \theta_{n-1}$ which cause manipulator joint axes $5, \cdots, n$ to be reciprocal to $\boldsymbol{8}_{R}^{*}$ can similarly be determined by recursion.

Remark: Genericly, for a given screw in $S$, there are $2^{n-2}$ configurations in which the joint axis screws are reciprocal to the given screw.

Since the singular configurations are independent of $\theta_{1}$ and $\theta_{n}$, and $S$ contains $\infty^{3}$ screws, the singularities of generic n-revolute-jointed manipulators form five-dimensional surfaces in the configuration space and works pace.

This method can numerically trace the critical point surfaces, like the algorithm in Section 3 . In this case, there are three sweeping parameters $h, \beta_{R}$, and $\alpha_{R}$. [10] develops another parametrization in $\theta_{2}, \theta_{3}$, and $\theta_{4}$. This algorithm is also subject to bifurcations. Analogously, (4.6) will have an extra branch of solutions when ${ }^{1} D_{1}={ }^{1} D_{2}={ }^{1} D_{3}=0$. A derivation similar to Section 3 shows that bifurcations occur when

$$
\frac{\partial G_{j}}{\partial \theta_{j+1}}=-{ }^{j} D_{1} \sin \theta_{j+1}+{ }^{j} D_{2} \cos \theta_{j+1}
$$

changes sign. The extra branches are traced in a manner similar to the procedure outlined in \$3. Similarly, this algorithm has initial condition bifurcations when: the first two axes are parallel; the first two axes are orthogonal; and the first two axes intersect. A more complete discussion of these conditions can be found in [10].

Since singular configurations are independent of $\theta_{1}$ and $\theta_{n}$, the loci of critical values related to a given singular screw form up to $2^{n-1} 2$-dimensional tori in $\mathbb{R}^{3}$. Figure 4 illustrates these tori for the singular configurations of a $6 R$ manipulator (the same as Fig. 2) which is reciprocal to a screw of pitch $h=1.0$. The singular screw axis is superimposed on the figure.

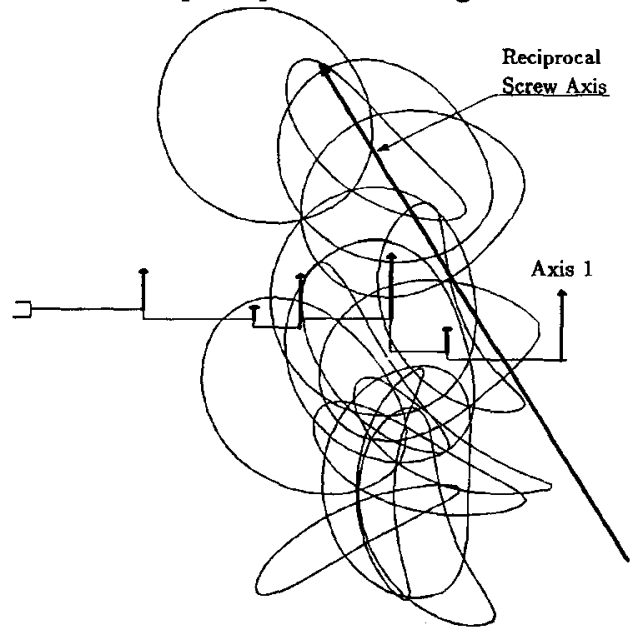

Figure 4: Cross Section of $6 R$ Manipulator Singular End-effector Locations for Singular Configurations which are Reciprocal to One Screw
Finally, [10] considers in more detail the special case of manipulators with wrists, where it is shown that:

Remark: The singular screw field of a spatial manipulator with wrist has $\infty^{3}$ screws which can be divided into two categories. (1) the wrist subchain is not singular, and the singular screw must have zero pitch and intersect the wrist concurrency point. These singularities can be analyzed using the methods of Sections 2,3. (2) the wrist subchain is singular, and the pitch of the singular screw can be finite or infinite.

\section{Conclusion}

This paper developed recursive algorithms for determining the singular set of spherical, regional, and spatial revolute-jointed manipulators with arbitrary numbers of joints and arbitrary geometries. The primary algorithm and its recursion was constructed by a geometric application of screw theory. This primary algorithm is directly applicable to manipulators with generic geometries. However, the critical point surfaces of non-generic manipulators bifurcate, and extensions of the algorithm to compute the extra bifurcation branches were developed.

\section{References}

[1] K. Sugimoto, J. Duffy, and K. H. Hunt, "Special Configurations of Spatial Mechanisms and Robot Arms," Mechanism and Machine Theory, vol. 17, no. 2, 1982, pp. 119-132.

[2] K. Sugimoto and J. Duffy, "Determination of Extreme Dire tances of a Robot Hand-Part1: A General Theory," Journal of Mechanical Design, Transaction of the ASME, vol. 103, July 1981, pp. 631-636.

[3] K. Sugimoto and J. Duffy, "Applications of Linear Algebra to Screw Systems," Mechanism and Machine Theory, vol. 17, no. 1, 1982, pp. 73-83.

[4] K.J. Waldron, S.L. Wang, and S.J. Bolin, "A Study of the Jacobian Matrix of Serial Manipulators," Journal of Mechanisms, Transmissions, and Automation in Design, vol. 107, June 1985, pp. 230-238.

[5] S.L. Wang and K.J. Waldron, "A Study of the Singular Configurations of Serial Manipulators," ASME Paper 86-DET-61.

[6] D. Kohli and M.S. Hau, "The Jacobian Analynis of Workspaces of Mechanical Manipulators," Mechanism and Machine Theory, vol. 22, no. 3, pp. 265-275, 1987.

[7] D. Kohli and M.S. Heu, "Boundary Surfaces and Accennibility Regions for Regional Structures of Manipulaton," Mechanism and Machine Theory, vol. 22, no. 3, pp. 277-289, 1987.

[8] D.K. Pai, "Singulnrity, Uncertainty, and Compliance of Robot Manipulators," Ph.D. Thesis, Dept. of Mechanical Engineering, Comell University, Ithaca, N.Y., May 1988.

[9] J.W. Burdick, "A Classification of 3R Regional Manipuletor Singularities and Geometries," Proc. IEEE Int. Conf. Robotics and Automation, Sacramento, CA, May, 1991.

[10] J.W. Burdick, "A Recurive Method for Finding RevolutoJointed Manipulator Singularities," Robotics and Mechanical Systems Report No. RMS-90-02, California Inetitute of Technology.

[11] H.B. Keller, Lectures on Numerical Methods in Bifurcation Problems Springer-Verlag, New York, 1987. 